\title{
Histoire et historiographie de la Kriminologie allemande : une introduction
}

Introduction to the History and Historiography of German Kriminologie

\section{Grégory Salle}

\section{OpenEdition}

12 Journals

\section{Édition électronique}

URL : http://journals.openedition.org/conflits/18924

DOI : $10.4000 /$ conflits. 18924

ISSN : $1777-5345$

Éditeur :

CCLS - Centre d'études sur les conflits lilberté et sécurité, L'Harmattan

\section{Édition imprimée}

Date de publication : 31 décembre 2014

Pagination : 241-251

ISBN : 978-2-343-05760-6

ISSN : 1157-996X

Référence électronique

Grégory Salle, " Histoire et historiographie de la Kriminologie allemande : une introduction », Cultures \& Conflits [En ligne], 94-95-96 | été-automne-hiver 2014, mis en ligne le 20 février 2016, consulté le 30 mars 2021. URL : http://journals.openedition.org/conflits/18924 ; DOI : https://doi.org/10.4000/ conflits. 18924 


\section{Histoire et historiographie de la Kriminologie allemande : une introduction}

\section{Grégory SALLE}

Grégory Salle est chargé de recherche au CNRS, rattaché au CLERSÉ (UMR 8019, Lille). Ses travaux ont jusqu'ici principalement porté sur les pratiques coercitives et les régulations normatives à l'aune du modèle juridico-politique de l' "État de droit ". Il a récemment publié "Statactivism against the penal machinery in the aftermath of "1968". The case of the French Groupe d'information sur les prisons ", Partecipazione e Conflitto. The Open Journal of Sociopolitical Studies, 7-2, 2014, pp. 221-236 et a coordonné en 2013, avec Gilles Chantraine, un dossier de la revue en ligne Champ Pénal/Penal Field (bttp://champpenal.revues.org/8380) sur la délinquance en col blanc.

Cur quoi peut-on appuyer la critique - au sens ordinaire comme au sens phiOlosophique - de la criminologie, allemande en l'espèce ? Autrement dit, quels sont les registres de discours, en particulier de discours à prétention scientifique, disponibles pour une telle critique ? On propose ici quelques éléments de réponse au moyen d'une introduction bibliographique, par définition succincte et partielle, relative à un domaine de recherche qui a connu un essor notable depuis le tout début des années 2000. Cette introduction espère faire mieux connaître un certain nombre de travaux germanophones et anglophones en la matière, sans pour autant avoir l'ambition ni la prétention de constituer une mise en perspective historiographique à l'instar de celles, fort utiles, de Herbert Reinke sur le crime et sa répression en général et de Falk Bretschneider sur l'enfermement en particulier ${ }^{1}$. Elle permet toutefois de passer en revue les principaux types d'approche ou angles d'attaque à ce sujet. Et

1. Reinke H., "Crime and criminal justice history in Germany. A report on recent trends", Crime, Histoire E Sociétés, 13-1, 2009, pp. 117-137 ; Bretschneider F., "Toujours une histoire à part ? L'état actuel de l'historiographie allemande sur l'enfermement aux XVIII' et XIXe siècles ", Crime, Histoire E Sociétés, 8-2, 2004, pp. 141-162. Ce dernier a par ailleurs dirigé l'ouvrage bilingue Der Kriminelle. Deutsch-französische Perspektiven/Le criminel. Perspectives franco-allemandes, Leipzig, Leipziger Universitätsverlag, 2007. 
d'en distinguer trois, qu'on pourra considérer, en dernière analyse, à l'aune du positionnement foucaldien en la matière.

Une première manière de nourrir cette critique consiste à puiser dans l'histoire de la criminologie, sans préjuger de l'opportunité ou non de consacrer un savoir autonome portant ce nom. En parcourant cette histoire, force est de constater qu'on est bien en peine de trouver un apport salutaire à mettre au crédit de cette discipline - reconnaissons-lui cette existence, académiquement consacrée en Allemagne et objectivée entre autres par des manuels ${ }^{2}$. Entendons : de cette discipline dès lors que ses porte-parole en assument sans états d'âme la dénomination. Les contributions les plus notables dans ce domaine proviennent en effet, au moins depuis la deuxième moitié des années 1960, d'auteurs mal à l'aise avec cette étiquette, soit qu'ils se présentent comme tenants d'une bien différente sociologie du crime [Kriminalsoziologie], soit qu'ils accolent au terme criminologie l'épithète "critique " [kritische kriminologsche] pour marquer leur distanciation, jusqu'à parler pour une poignée d'entre eux, à l'instar du regretté Stanley Cohen, d'anticriminologie [antikriminologie ${ }^{3}$. Il faut dire que jusqu'alors, l'histoire de la Kriminologie allemande (ouest-allemande en l'occurrence) était si peu reluisante qu'il était tout sauf difficile de trouver de quoi la déconsidérer. C'est une histoire faite pour l'essentiel de stigmatisation et d'hygiénisme puis d'eugénisme, peuplée d'individus dégénérés et autres êtres de mauvais aloi [Minderwertigen], corrompus par des tares congénitales et des dérèglements héréditaires, décrétés incorrigibles si besoin, à enfermer sinon à éliminer. Une histoire pathologisante (parce que biologisante et/ou psychologisante), conservatrice le plus souvent, au service d'une défense tantôt fébrile, tantôt agressive - et le cas échéant meurtrière - de l'ordre établi, les voies alternatives ayant presque systématiquement été obturées.

Il est tentant, pour clore d'emblée - sans doute un peu trop facilement - le chapitre, de se situer directement au cœur du pire, aux « heures les plus sombres » de la criminologie, c'est-à-dire évidemment durant le nazisme ${ }^{4}$. Il faut avoir un talent certain dans le rôle de l'avocat du diable pour chercher alors la nuance, la complexité, l'ambiguïté. Mais on ne saurait nullement envisager le rôle endossé par la criminologie pendant cette période comme une funeste parenthèse. La stérilisation ou l'euthanasie forcée des « asociaux » héréditaires

2. Sur ces manuels, voir Schneider H-J., « Die deutschsprachige Kriminologie der Gegenwart. Kritische Analyse anhand deutschsprachiger kriminologischer Lehrbücher », in Liebl K. (ed.), Kriminologie im 21. Jahrhundert, Wiesbaden, VS Verlag für Sozialwissenchaften, 2007, pp. 145-174. Voir plus largement cet ouvrage collectif pour un état des lieux récent, faisant part d'un désenchantement par comparaison avec l'« âge d'or » (sociologique) des années 1970.

3. Décédé début 2013, Stanley Cohen fut entre autres l'auteur en 1988 d'un livre intitulé Against Criminology (New Brunswick, Transaction Books).

4. Rafter N., "Criminology's Darkest Hour: Biocriminology in Nazi Germany", The Australian and New Zealand Journal of Criminology, 41-2, 2008, pp. 287-306. Cet article de synthèse esquisse au passage une comparaison avec le cas de l'Italie fasciste. 
s'est appuyée sur les théories biologiques du crime qui avaient acquis une position dominante dès l'institutionnalisation de la criminologie au cours du dernier quart du siècle précédent. Leur rôle dans l'extermination de masse fut au moins - quand il ne fut pas direct - celui de la justification savante, animée par un scientisme aussi aveugle qu'erroné. Ainsi, en choisissant la période du Troisième Reich, en tant que telle mais aussi en tant qu'elle dérive de l'état des rapports de forces ayant animé les périodes antérieures, il est aisé de jeter le discrédit sur une criminologie qui offrait, au moins dans sa version largement dominante, une prise facile au pouvoir nazi. Et ceci même en restant attentif au piège du regard téléologique (une mesure spécifique, comme l'internement préventif des malades mentaux considérés comme dangereux, n’a pas une signification identique selon le système d'idées et de pratiques dans lequel elle s'inscrit) et en dépit parfois d'une cruelle ironie - ainsi le psychiatre Gustav Aschaffenburg (1866-1944), figure clé au début du XXe siècle du développement d'une biologie criminelle instrumentalisée par la suite, était-il juif et dut émigrer aux États-Unis pour fuir les persécutions antisémites.

C'est dire que la période antérieure au nazisme est, sur le plan historiographique, tout aussi digne d'intérêt. Si, au cours de l'époque wilhelminienne, domine et même triomphe une approche biologisante et médicalisante ${ }^{5}$, il reste que si l'on s'efforce de la considérer sous toutes ses facettes, la criminologie désigne un ensemble qui n'est pas unitaire et demeure tiraillé par des rapports de forces entre doctrines, disciplines, courants, postulats. Ce qui pousse alors à en explorer les différentes facettes plutôt que de la saisir en bloc (et de la condamner comme telle).

Lisant la période nazie à la lumière des décennies précédentes, l'historien Richard Wetzell s'est ainsi efforcé de naviguer entre deux écueils symétriques. D'un côté, l'apologie en creux d'une science pure en soi, qui aurait été dévoyée et dénaturée par une dérive aberrante. De l'autre, la dénonciation d'une science promise d'avance à se mettre au service d'une entreprise sanglante. On doit à cet historien états-unien la première étude systématique sur la genèse et le développement de la criminologie allemande couvrant la période allant du Kaiserreich à la fin du nazisme. Son ouvrage Inventing the Criminal ${ }^{6}$ retrace l'avènement d'une discipline marquée par la victoire des approches médicales sur les approches sociales et sociologiques - idée générale que sont venus depuis confirmer des travaux récents ${ }^{7}$. La période 1880-1914 est le théâtre

5. Wetzell R. F., "The Medicalization of Criminal Law Reform in Imperial Germany", in Finzsch N., Jütte R. (eds.), Institutions of Confinement, Cambridge University Press, 1997, pp. 275-284.

6. Wetzell R. F., Inventing the Criminal. A History of German Criminology (1880-1945), Chapel Hill/London, University of North Carolina Press, 2000. À l'heure où j'écris ces lignes paraît un ouvrage collectif sous sa direction, Crime and Criminal Justice in Modern Germany (Berghahn Books, 2014) qu'il n'est pas possible de prendre en compte ici.

7. Voir par exemple Schauz D., Freitag S., «Verbrecher im Visier der Experten. Zur Einführung ", in Schauz D., Freitag S. (eds.), Verbrecher im Visier der Experten. 
d'une défaite cuisante, chez les partisans d'une réforme juridique du système pénal, des approches sociologiques - a fortiori des réflexions autour de ce qu'on appellerait aujourd'hui la construction sociale du crime (enjeux de délimitation, de qualification, de hiérarchisation des infractions, etc.) - au profit des approches médicales et particulièrement psychiatriques, selon une logique qui n'est pas sans rappeler celle de la France ${ }^{8}$. Ce succès tient notamment au fait que, contrairement à la sociologie, ces savoirs promettent des solutions concrètes en matière de politique étatique. L'auteur signale néanmoins l'ambivalence des implications politiques de la criminologie, dont le courant dominant, réactionnaire et répressif, n'excluait pas des usages minoritaires potentiellement progressistes ou humanitaires. Il prend garde d'indiquer que certains criminologues relativement éminents étaient « libéraux » et non protonazis. Il rappelle ainsi que Franz von Liszt (1851-1919), figure centrale de la criminologie définie comme savoir de gouvernement au service de la défense sociale, en réaction et en guise d'alternative à la pensée pénale dissuasive et rétributive classique, fut au début du siècle député du "parti progressiste », le classant alors comme un «libéral de gauche " [left-liberal] - classement au demeurant discutable 9 . On peut ajouter que si Liszt a fait l'objet ultérieurement d'une lecture "progressiste » à partir de l'une de ses formules répétée à l'envi (et simplifiée à l'excès) selon laquelle il n'y a de bonne politique pénale qu'une politique sociale, à partir aussi de l'idée que la peine doit se concevoir comme un traitement et non seulement une punition, une telle lecture procède d'un sérieux inventaire et ne reflète nullement l'ensemble de l'œuvre de Liszt, ni même l'esprit dans lequel cet énoncé fut exprimé. La formule en question était pour le moins équivoque : une politique «sociale », qualificatif polysémique et flou par excellence, n'est pas en soi positive et peut signifier le meilleur comme le pire. En outre, Liszt n'a jamais travaillé à la réforme sociale et s'est toujours cantonné à l'individu criminel, tout particulièrement aux criminels dits d'habitude, jugés dégénérés par l'hérédité et/ou par l'influence corruptrice de leur milieu. S'il n'est pas forcément question chez lui de tares congénitales, il l'est au moins de prédispositions pathologiques naturelles. La politique sociale consiste alors essentiellement en une extension du contrôle social vouée à repérer plus efficacement les dégénérés, si possible avant même qu'ils aient commis un crime. Dès les années 1890, dans le cadre de l'essor d'une pensée eugéniste, les partisans d'une stérilisation des déficients mentaux

Kriminalpolitik zwischen Wissenschaft und Praxis im 19. und frühen 20. Jabrbundert, Stuttgart, Franz Steiner Verlag, 2007, pp. 9-32, le livre de Silviana Galassi dont il sera question infra, ou l'ouvrage de Christian Müller (auquel je n'ai pas pu me rapporter pour le présent texte) Verbrechensbekämpfung im Anstaltsstaat: Psychiatrie, Kriminologie und Strafrechtsform in Deutschland. 1871-1933, Göttingen, Vandenhoeck \& Ruprecht, 2004.

8. Cf. Mucchielli L., "Criminologie, hygiénisme et eugénisme en France (1870-1914) : débats médicaux sur l'élimination des criminels réputés “incorrigibles" ", Revne d'histoire des sciences humaines, 3, 2000, pp. 57-88.

9. On peut plutôt, comme y invite Gilbert Badia (Les Spartakistes. 1918, l'Allemagne en révolution, Bruxelles, Aden, 2008, p. 4) placer ce parti, représentant de la moyenne bourgeoisie, au centre de l'échiquier politique. 
à des fins d'hygiène sociale se font entendre. Les fondements - et même plus que les fondements - d'un discours sur la pureté de la race sont alors posés.

Le tableau est bien différent de celui observable au milieu du XIXe siècle, dépeint par l'autre historien à avoir rouvert ce champ d'étude, Peter Becker ${ }^{10}$, avec lequel $\mathrm{R}$. Wetzell a du reste coordonné en 2006 un volume collectif offrant des perspectives comparatives ${ }^{11}$. Le triomphe du regard médical à la fin du siècle apparaît d'autant plus frappant à la lumière de cette période pour ainsi dire préhistorique. P. Becker décrit en effet la criminologie d'avant la criminologie, quand celle-ci se confond encore plus ou moins avec la Kriminalistik (art pratique, empirique au premier sens du terme, sur laquelle il a également écrit ${ }^{12}$ ), c'est-à-dire quand elle est incarnée par une constellation de médecins, philanthropes, magistrats, policiers, statisticiens, théologiens... Bref, par des praticiens et non (seulement) des savants, à un moment où l'autorité en la matière provient d'une expérience personnelle située plus que d'un savoir constitué-institué. L'intérêt du travail de P. Becker, qui s'appuie sur une vaste palette de sources écrites (y compris non imprimées), est de cartographier cet ensemble relativement hétérogène en définissant la criminologie en un sens large, allant d'idées théoriques à des préceptes à destination des gouvernants, en passant par des pratiques professionnelles. L'auteur montre que les représentations des criminels, lesquels sont définis par opposition au modèle bourgeois (les amalgames entre les figures du criminel et du révolutionnaire étant courants), mettent pour l'heure l'accent sur l'infériorité morale, sur la déchéance morale de l'homme qui manque d'autocontrôle, plus que sur l'infériorité physique, en dépit de la popularité de la théorie de la dégénération. En particulier, il met en relief deux types de récits autour de deux figures typiques [Erzäblmuster] : d'une part celle de l'homme déchu [gefallenen Mensch], envisagée sous un versant moral/éthique, soit l'homme victime pour partie de ses propres incapacités, de mauvais exemples aussi, défini donc par ses caractéristiques biographiques; d'autre part, sur un versant cette fois biomédical, celle de l'homme empêché ou entravé [verbinderten Mensch], défini par ses (prétendus) traits anthropologiques et bientôt anthropomorphiques.

Si l'on se projette quelques décennies plus tard, le « kaléidoscope weimarien » 13 apparâit à l'évidence comme un autre moment clé ${ }^{14}$. Ambivalente

10. Becker P., Verderbnis und Entartung: eine Geschichte der Kriminologie des 19. Jabrhunderts als Diskurs und Praxis, Göttingen, Vandenhoeck \& Ruprecht, 2002, notamment pp. 365-371.

11. Becker P., Wetzell R. F. (eds.), Criminals and Their Scientists: The History of Criminology in International Perspective, Cambridge/New York, Cambridge University Press, 2006. Le volume permet de nourrir la comparaison franco-allemande, grâce aux contributions de Marc Renneville, Philippe Artières, Martine Kaluszynski et Laurent Mucchielli.

12. Voir Becker P., Dem Täter auf der Spur. Eine Geschichte der Kriminalistik, Darmstadt, Primus, 2005. Un parallèle intéressant avec la France, en mettant en perspective l'ensemble du $\mathrm{XIX}^{\mathrm{e}}$ siècle, serait là encore possible : $c f$. Mucchielli L., «Introduction générale. Naissance de la criminologie », in Histoire de la criminologie française, Paris, L'Harmattan, 1994, pp. 7-18. 
entre toutes, la période est le théâtre d'une radicalisation progressive du thème des « asociaux » 15 et de la manière de se débarrasser des êtres jugés inférieurs et nuisibles, sur fond de réduction des prestations sociales accordées aux populations les plus vulnérables. On peut évoquer ici un point signalé par plusieurs auteurs, mais sur lequel une étude systématique reste semble-t-il à mener ${ }^{16}$ : ce thème qu'on pourrait croire réservé aux organisations conservatrices est en fait courant, y compris à gauche de l'échiquier politique. Comme le remarque incidemment Richard J. Evans à la fin de sa plongée dans le German underworld - livre dans lequel il replace la vision médicale dans l'histoire des perceptions relatives aux déviants et aux marginaux au cours du XIX siècle en évoquant au passage la prégnance du darwinisme social en Allemagne par rapport à d'autres pays européens ${ }^{17}$ - on ne compte pas de mobilisation révolutionnaire autour du crime, car les socialistes méprisent aussi les actes illégaux d'un sous-prolétariat jugé versatile et périlleux dans la tradition marxiste. Si le SPD souligne le rôle de la pauvreté et dénonce une justice de classe, ses porte-parole n'en partagent pas moins une bonne part des préjugés du courant majoritaire et ne sont pas insensibles à la séduction de l'approche génétique et plus largement biomédicale pour séparer le peuple et la plèbe. Autrement dit, le triomphe de cette vision prospère aussi sur la faiblesse intellectuelle et institutionnelle des visions alternatives.

Par rapport aux périodes abordées brièvement jusqu'ici, celle de l'immédiat après-guerre pourrait a priori paraître d'un moindre intérêt. Pourtant, si elle est certainement moins brûlante, elle n'en est pas moins révélatrice de ce qu'il devient difficile de ne pas qualifier de tropisme conservateur de la Kriminologie. Imanuel Bauman montre en effet, à partir du cas de la délinquance juvénile sur la période 1945-1968 mais aussi plus largement ${ }^{18}$, que

13. Je reprends cette expression à Solchany J., L'Allemagne au XXe siècle, Paris, PUF, 2003, pp. 81 sq.

14. Sur la période weimarienne (et la période nazie), voir en premier lieu Wetzell R. F., "Criminology in Weimar and Nazi Germany", in Becker P., Wetzell R. F. (eds.), Criminals and Their Scientists..., op. cit., pp. 401-423.

15. Voir en français Korzilius S., "Evolution de la thématique des "asociaux" dans la discussion sur le droit pénal pendant la République de Weimar », Astérion, 4, 2006, pp. 45-72 (en ligne, URL : http://asterion.revues.org/511).

16. D'après le compte-rendu d'une journée d'étude ayant eu lieu en 2001 sur le thème "Crime and Criminal Justice in Modern Germany, 1870-1960” (GHI Bulletin, 29, automne 2001, pp. 75-76), la question semble toutefois avoir été travaillée par Andreas Fleiter dans le cadre d'une thèse réalisée à l'université de Bochum, intitulée Straf-und Gefängnisreformen in Deutschland und den USA : Preußen und Maryland, 1870-1935.

17. Evans Richard J., Tales from the German Underworld. Crime and Punishment in the 19th Century, New Haven, Yale University Press, 1998, pp. 215-221. Suggestion similaire chez Wachsmann N., Hitler's Prisons. Legal Terror in Nazi Germany, New Haven, Yale University Press, 2004, p. 48.

18. Bauman I., "Interpretation und Sanktionierung von Jugendkriminalität », in Herbert U. (ed.), Wandlungsprozesse in Westdeutschland: Belastung, Integration, Liberalisierung, 19451980, Göttingen, Wallstein, 2002, pp. 348-379. C'est aussi un argument central de son ouvrage Dem Verbrecher auf dem Spur. Eine Geschichte der Kriminologie und Kriminalpolitik in Deutschland 1880 bis 1980 (Göttingen, Wallstein, 2006) qui couvre le XXe siècle ouest-allemand. 
1945 ne constitue pas une césure du point de vue des représentations savantes du crime et des criminels. En dépit d'un discrédit cinglant, bien des continuités règnent en la matière. Les conceptions héréditaires basées sur l'idée de dispositions innées ou endogènes de certains jeunes corrompus ou de mauvaise composition [Minderwertigkeit] restent largement répandues dans des travaux « renommés » de la criminologie des années 1950, laquelle s’inscrit sans grand changement (ni scrupule) dans la tradition antérieure. D’ailleurs, contre l'idée d'une conversion sociologique massive de la criminologie au cours de la décennie suivante, l'auteur de Dem Verbrecher auf dem Spur défend la thèse selon laquelle, en dépit de renouvellements marginaux (comme l'effort pour livrer des explications « multifactorielles ») et d'une perte d'influence certaine, la criminologie traditionaliste reste dominante sinon hégémonique à la fin des années 1960. En travaillant sur les réformes pénales qui débutent laborieusement dans les années 1950, Petra Gödecke a confirmé le fait que seule une petite minorité de juristes se prononce alors en faveur d'une peine orientée vers la réhabilitation des anciens détenus, la majorité demeurant fidèle à une conception neutralisante et rétributive de la peine solidaire des idées héritées sur le crime et les criminels ${ }^{19}$.

Bref, même à l'observation de la période en quelque sorte la plus « favorable », après une disqualification qui aurait dû déboucher sur une redéfinition intégrale, le moins que l'on puisse dire est que le mainstream criminologique ne compte pas parmi les moments les plus glorieux de l'histoire des sciences.

L'histoire des sciences, c'est précisément ce qui nourrit une deuxième manière de critiquer la criminologie. Cette deuxième manière n'est plus seulement d'ordre historique ; elle est à cheval entre l'histoire et l'épistémologie. Il s'agit alors de dénier à la criminologie le statut de science sérieuse, robuste bref : accomplie - et accréditée à siéger parmi les savoirs respectables.

C'est l'essentiel de la démonstration de Silviana Galassi 20. Si le titre de son livre annonce le sujet et la période (la criminologie sous l'Empire), du moins en partie (le pari est en effet d'appréhender simultanément la criminologie et le mouvement de réforme pénale), c'est le sous-titre qui en donne la clef de lecture. Il s'agit de l'histoire (sociale) d'une « scientifisation »- le terme fait l'objet d'une longue explicitation en introduction - interrompue, brisée, et partant inachevée, avortée. C'est la constitution de cet argument en ligne directrice qui fait l'originalité de l'ouvrage, plus que l'évolution historique que décrit ce dernier. Les conditions intellectuelles de constitution de la criminologie comme savoir empirique particulier (à commencer par la conversion du

19. Gödecke P., «Die Strafrechtsreform zwischen Vergeltung und Resozialisierung, rigider "Sittlichkeit" und vorsichtiger Toleranz », in Requate J. (ed.), Recht und Justiz in gesellschaftlichen Aufbruch (1960-1975), Baden-Baden, Nomos, 2003, pp. 261-273.

20. Galassi S., Kriminologie im Deutschen Kaiserreich. Geschichte einer gebrochenen Verwissenschaftlichung, Stuttgart, Franz Steiner, 2004. 
regard que suppose le fait même de poser un regard à prétention scientifique sur le «crime », comme phénomène empirique sur lequel il est possible d'agir, de même que sur le « criminel », comme être qu'il serait possible de repérer) qu'elle rapporte, aussi bien que le contexte social et politique général qui en fournit le cadre (comme les craintes de la bourgeoisie à l'égard d'un prolétariat d'autant plus menaçant qu'il s'organise, dont il est donc profitable de dramatiser la criminalité, si besoin en interprétant les statistiques dans le sens souhaité), étaient pour l'essentiel connus, même si de manière parfois éparse, grâce aux travaux que nous avons déjà cités (et auxquels d'ailleurs elle ne se réfère guère). Certes, l'auteure agrandit et affine parfois le tableau en détaillant certains aspects, concernant les luttes entre courants et l'articulation entre savoir criminologique et politique pénale, ou en insistant sur le mélange entre pessimisme culturel et optimisme scientiste. Mais surtout elle systématise, en historienne et non en criminologue, un argument qui n'est qu'esquissé ou timidement avancé chez d'autres auteurs : cette "scientifisation » incomplète, et finalement ce statut épistémologiquement fragile, de la criminologie.

Cette imperfection traverse les deux phases du processus, que S. Galassi distingue sous l'influence entre autres de Max Weber (p. 16-24) : une scientifisation primaire, qui concerne la discipline elle-même, et une autre secondaire, qui concerne son institution et sa diffusion dans la société. Sur ces deux plans, explique l'auteure, la criminologie - si tant est qu'on puisse en parler au singulier - a échoué. Ce seuil de scientificité, elle ne l'a pas franchi. Somme toute, la criminologie a surtout consisté en un enrobage savant de conceptions normatives et de préjugés sociaux ${ }^{21}$. De nos jours d'ailleurs, en dépit de sa reconnaissance institutionnelle, les contours et la consistance de la Kriminologie demeurent imprécis et elle n'est aucunement un domaine unifié. Le livre n'est pas sans susciter la critique ; il procède notamment d'une conception de la science qui paraît quelque peu datée. Néanmoins, la description approfondie qu'il renferme achève de convaincre que prendre ses distances à l'égard de la criminologie en tant que telle n'a rien d'une position farfelue.

Car ne nous y trompons pas : la fragilité épistémologique de la criminologie, qui d'ailleurs n'a jamais caché dès sa genèse sa vocation d'être une science de gouvernement, n’empêche pas que ce « savoir spécial », dont P. Pasquino a bien résumé les conditions intellectuelles d'émergence ${ }^{22}$, procède d'une vraie rupture, d'un changement de paradigme.

De là, on peut être amené à une troisième approche critique, d'ordre cette fois théorico-épistémologique ou conceptuel. Dans cette perspective, la voie

21. Toujours pour alimenter la comparaison, voir la « mise en perspective épistémologique de la criminologie » effectuée par Laurent Mucchielli in Histoire de la criminologie française, op. cit., pp. 453-505.

22. Pasquino P., «Naissance d'un savoir spécial : la criminologie », Sociétés E représentations, 3, 1996, pp. 173-186. 
historique dominante suivie par la criminologie au cours de son développement, et la défaite systématique de l'approche sociologique à laquelle elle a correspondu, ne sont pas contingents. Elles dérivent d'une constitution disciplinaire d'emblée problématique, du fait que le nom même de criminologie évoque et implique toujours tendanciellement une conception substantialiste de son objet ; qu'en tout cas elle comporte en permanence ce risque. Poussé jusqu'à son terme, ce raisonnement est nominaliste. Il réfute ou, du moins, repousse l'idée - même s'il peut la constater empiriquement, puisqu'il existe de fait, dans plusieurs pays, des auteurs «progressistes » acceptant la dénomination de «criminologues »- selon laquelle cette dénomination disciplinaire ne serait au fond qu'une étiquette ne prêtant pas à conséquence, sur le mode $\mathrm{du}$ « qu'importe le flacon, pourvu qu'on ait l'ivresse ». En un mot, il considère que la «criminologie » en tant que telle est nulle et non avenue. Pourquoi ? Parce que le crime, comme phénomène social, ne peut être extrait de l'ensemble des relations sociales, au même titre par exemple que le suicide ou le mariage et que, dès lors, une "criminologie » n'a pas plus de pertinence qu'une «suicidologie» ou une «mariagologie». Difficile de trouver une expression plus claire de cette position que celle-ci :

\begin{abstract}
"Autant dire que pour nous il ne pent y avoir de "bonne criminologie". Humanitaire ou personnaliste, critique ou radicale, et quel que soit le travesti qu'elle emprunte (droit, psychologie, sociologie...), la criminologie, en tant que "science ayant pour objet d'étude spécifique le crime et la délinquance”, est - et ne peut qu'être - une discipline (au double sens du terme) répressive. Cela dans la mesure où elle tend à diffuser et à légitimer par des arguments d'apparence rationnelle (statistiques, théories...) les mécanismes de répression propres à tout système de domination $» 23$.
\end{abstract}

On monte ici d'un cran : ce n'est pas seulement que la criminologie est une science de gouvernement - cela elle le dit elle-même, elle l'assume ouvertement -, c'est qu'elle est, qu'on le veuille ou non, une science conservatrice de légitimation/protection de l'ordre établi, ce que d'ailleurs ses fondateurs disaient presque aussi clairement ${ }^{24}$.

Sans doute une telle conception nominaliste est-elle davantage ancrée en France où la sociologie, avec Tarde puis Durkheim, a en quelque sorte remporté la mise face à la criminologie, en dépit de l'« échec relatif de la sociologie durkheimienne » 25 . Encore cette conception a-t-elle été certainement en perte

23. Actes. Cabiers d'action juridique, Délinquances et ordre, Paris, François Maspero, 1978, p. 17.

24. Il n'est qu'à lire pour s'en convaincre la conférence d'ouverture du cours de droit pénal faite le 27 octobre 1899 par Franz von Liszt à l'université de Berlin, traduite en français sous le titre L'objet et la méthode des sciences pénales, Paris, Arthur Rousseau, 1902.

25. Voir Mucchielli L., La déconverte du social. Naissance de la sociologie en France (1870-1914), Paris, La Découverte, 1998, chap. 8. 
de vitesse depuis l'époque de la citation qui précède, des formulations aussi tranchées paraissant plus difficiles à trouver dans la période immédiatement contemporaine. Quoiqu'il en soit, en Allemagne, la criminologie dite critique oscille entre cette position nominaliste - selon l'idée que la criminologie ne comporte pas d'erreurs, mais qu'elle est elle-même une erreur ${ }^{26}$ - et une position qu'on peut dire pragmatique, ne s'attachant qu'au signifié du signifiant «criminologie » (en d'autres termes, au contenu dont il est investi ou aux usages dont il fait l'objet) et se méfiant du fétichisme terminologique auquel risque selon elle d'aboutir la position nominaliste ${ }^{27}$. C'est la seconde option qui, au cours des dernières décennies, a pris l'ascendant sur la première ; les choses ne sont cependant pas jouées une fois pour toutes.

Ce tour d'horizon, nous l'avons dit d'emblée, n'était précisément qu'un tour d'horizon, limité qui plus est à la République fédérale (laissant donc de côté le cas de la RDA). L'effort de synthèse ne rend sans doute pas justice à la richesse des travaux mentionnés. De plus, des pans tout à fait importants n'ont pas été abordés ici, comme l'objectivation des femmes par le discours criminologique et donc la dimension de genre ${ }^{28}$. Pour qui voudrait aller plus loin, cette question, ainsi que d'autres (par exemple celle des rapports entre criminologie et institutions d'assistance et d'encadrement, ou celle du rôle de l'essor du calcul statistique dans la constitution de la discipline criminologique), sont traitées dans un important ouvrage collectif dirigé par Désirée Schauz et Sabine Freitag, dont les contributions s'efforcent généralement de replacer la criminologie dans un contexte, notamment institutionnel, plus large 29.

Revenons à présent à notre questionnement initial. «Vous avez lu quelquefois des textes de criminologues ? C'est à vous couper bras et jambes. Et je le dis avec étonnement, non avec agressivité, parce que je n'arrive pas à comprendre comment ce discours de la criminologie a pu en rester là. On a l'impression que le discours de la criminologie a une telle utilité, est appelé si fortement et rendu si nécessaire par le fonctionnement du système qu'il n'a même pas eu besoin de se donner une justification théorique, ou même simplement une cohérence ou une armature. Il est entièrement utilitaire. » 30 À lire trop rapidement cet extrait, ou au souvenir des railleries contre le « bavardage de la

26. Voir Steinert H., «"Die Kriminologie hat keine Fehler, sie ist der Fehler" : kulturindustrielles Wissen über Kriminalität und populistische Politik ", Jabrbuch für Rechts- und Kriminalsoziologie '04, 2005, pp. 265-279.

27. Typique de cette oscillation, la conclusion de Cremer-Schäfer H., Steinert H., Straflust und Repression. Zur Kritik der populistischen Kriminologie, Münster, Westfälisches Dampfboot, 1998, p. 249.

28. Voir Uhl K., Das "verbrecherische Weib". Geschlecht, Verbrechen und Strafen im kriminologischen Diskurs, 1800-1945, Berlin/Münster, LIT Verlag, 2003

29. Schauz D., Freitag S. (eds.), Verbrecher im Visier der Experten. Kriminalpolitik zwischen Wissenschaft und Praxis im 19. und früben 20. Jabrbundert, Stuttgart, Franz Steiner Verlag, 2007. 
criminologie » 31 , on pourrait être tenté de réduire les critiques formulées par Michel Foucault à l'encontre de la discipline criminologique à une critique d'ordre essentiellement, voire exclusivement, épistémologique. En réalité sa position, indissociablement épistémologique, politique et éthique (la méthode généalogique fournissant un socle à l'ensemble) est beaucoup plus ample et, disons-le, beaucoup plus intéressante; elle ne prend pleinement son sens que dans le cadre d'une critique générale des rationalités pénales, comme d'ailleurs dans celui d'une critique du discours des sciences humaines lui-même ${ }^{32}$.

Cette critique multidimensionnelle, pour ambitieuse qu'elle soit, est sans doute la seule réellement cohérente : elle évite aussi bien le déni du conflit de valeurs que l'énonciation d'une critique qui serait formulée depuis un poste épistémique imprenable. Elle est en tout cas appelée par la recrudescence d'un discours criminologique qui, sous des dehors en partie rénovés, renoue, parfois d'une façon si explicite et assumée qu'elle en devient confondante, avec le pire des discours du XIXe siècle. Les aperçus états-uniens de l'alliance entre droit pénal et neurosciences font à cet égard froid dans le dos ${ }^{33}$. On aurait sans doute tort de croire que l'Allemagne, par son histoire, est immunisée contre les tentations d'importation. Ceci d'autant que jusqu'ici, contrairement à une croyance assez répandue, l'autonomisation institutionnelle de la criminologie allemande, qui demeure sous la tutelle du droit pénal, est pour l'essentiel un échec ${ }^{34}$.

30. Foucault M., «Entretien sur la prison : le livre et sa méthode » (1975), Dits et Écrits, tome I (1954-1975), Paris, Gallimard, 2001, p. 1616.

31. Foucault M., Surveiller et punir. Naissance de la prison, Paris, Gallimard, 1993 [1975], p. 355. Il a d'ailleurs (faussement ?) concédé ultérieurement que le mot était « peut-être [...] un peu désinvolte » et proposé de le retirer («Qu'appelle-t-on punir ? » [1983-1984], Dits E Écrits, tome II (1976-1988), Paris, Gallimard, 2001, p. 1457).

32. Je renvoie à l'article de Fabien Gouriou et Olivier Razac dans ce numéro.

33. Voir Pustilnik A., "La violence dans le cerveau. Une critique de la contribution des neurosciences au droit criminel », in Ambroise B., Chauviré C. (eds.), Le mental et le social, Paris, EHESS, 2013, pp. 155-188.

34. Voir par exemple Oberwittler D., Höfer S., "Crime and Justice in Germany: An Analysis of Recent Trends and Research”, European Journal of Criminology, 2-4, 2005, pp. 480-481. Les auteurs renvoient à Karstedt S., "Standortprobleme: Kriminalsoziologie in Deutschland ». Soziologische Revue, 23, 2000, pp. 141-151. 